\section{Getty pilot project explores use of digifal images}

The Getty Art History Information Program (AHIP) and MUSE Educational Media have selected six museums and seven universities to participate in a pilot project to test the distribution of art images and information. Participating institutions will resolve issues of intellectual property rights, network security, and information standards, defining the terms and conditions for the educational use of museum images and information on campus networks. This Museum Educational Site Licensing Project runs from January 1, 1995, through June 30, 1997, and makes digitized images and descriptive texts representing at least 3,000 works of art (a minimum of 500 from each participating museum) available on the campus networks of participating universities without site license or royalty fees during the 1995-96 academic year. Information on and images of 3,000 more works will be added during 1996-97. Participating universities are: American University; Columbia University; Cornell University; University of Illinois at Urbana-Champaign; University of Maryland at College Park; University of Michigan at Ann Arbor, Dearborn, and Flint; and the University of Virginia, Charlottesville.

\section{French cave paintings online}

Images from the recent Combe d'Arc (Arde'che) prehistoric cave painting discovery are already on the Internet at the World Wide Web address: http://www.culture.fr/gvpda.htm. This link on the French Ministry of Culture's homepage leads to text and four images. As Jack Kessler reports in his newsletter FYIFrance, there are two remarkable things about this resource. First, it is a significant event in art history, and second, "thanks to networking's wonders it has been only a month since the cave paintings were found and suddenly everyone

\title{
SAA's Preservation Management Training Program is a huge success
}

December 1994 marked the close of a truly pathbreaking educational initiative, the Society of American Archivists' (SAA) Preservation Management Training Program. In 1991 the SAA received a grant worth about $\$ 650,000$ from the National Endowment for the Humanities' Division of Preservation and Access to launch the SAA Preservation Management Training Program. It was a three-year nationwide program that trained 44 archival administrators in establishing and maintaining comprehensive archival preservation management programs. The program was conducted through four regional series, Northeastern, Midwestern, Western, and Southeastern. Each series met independently for three one-week institutes spread across a one-year period. Its audience was mid-career archival administrators targeted to integrate preservation administration into their management perspectives. Participants were selected competitively within their region according to the strength of their application.

The program's pioneering aspects will profoundly effect the future of preservation education and training in libraries and archives. Its underlying philosophy addressed moving archival preservation away from ad hoc decisions based on restoring documents in an advanced stage of deterioration to well-planned management strategies aimed at preventing deterioration across a repository's entire holdings.

The curriculum advocated integrating preservation administration into all facets of the management of archives, not necessarily operating as a separate functional element in the same way as reference or collection development. The program's assignments were also exceptional because they were designed to build elements of a functioning, tailor-made archival preservation program for the student's employing institution prior to graduation.

Another unique characteristic was the program faculty's work with a professional educator/training expert to develop modern instructional methods based on interactive adult learning strategies that have been successfully applied by educators and trainers elsewhere. The SAA Preservation Management Training Program leaves us with a legacy of being the nation's most ambitious preservation education program undertaken to date.-Tyler $O$. Walters, Iowa State University 